\title{
BMJ Open Combination adherence strategy to support HIV antiretroviral therapy and pre-exposure prophylaxis adherence during pregnancy and breastfeeding: protocol for a pair of pilot randomised trials
}

\author{
Friday Saidi (D) , ${ }^{1}$ Wilbroad Mutale (D) , ${ }^{2}$ Kellie Freeborn, ${ }^{3}$ Nora E Rosenberg, \\ Lauren Aiko Graybill, ${ }^{5}$ Suzanne Maman, ${ }^{4}$ K. Rivet Amico, ${ }^{6}$ Katie R Mollan, ${ }^{5,7}$ \\ Twambilile Phanga, ${ }^{1}$ Beteniko Milala, ${ }^{1}$ Lauren M Hill, ${ }^{4}$ Allison M Gottwalt, ${ }^{3}$ \\ Sam Phiri, ${ }^{8,9,10,11}$ Thoko Kalua, ${ }^{12,13}$ Benjamin H Chi ${ }^{3}{ }^{3}$
}

To cite: Saidi F, Mutale W, Freeborn $\mathrm{K}$, et al. Combination adherence strategy to support HIV antiretroviral therapy and pre-exposure prophylaxis adherence during pregnancy and breastfeeding: protocol for a pair of pilot randomised trials. BMJ Open 2021;11:e046032. doi:10.1136/ bmjopen-2020-046032

- Prepublication history and additional supplemental material for this paper are available online. To view these files, please visit the journal online (http://dx.doi.org/10.1136/ bmjopen-2020-046032).

Received 19 0ctober 2020 Accepted 11 June 2021

Check for updates

(C) Author(s) (or their employer(s)) 2021. Re-use permitted under CC BY-NC. No commercial re-use. See rights and permissions. Published by BMJ.

For numbered affiliations see end of article.

Correspondence to

Dr Friday Saidi;

fsaidi@unclilongwe.org

\section{ABSTRACT}

Introduction To realise the expected gains from prevention of mother-to-child HIV transmission initiatives, adherence to preventative and therapeutic antiretroviral regimens is critical and interventions deployable in busy programmatic settings with a high HIV burden are needed. Based on formative research, we developed an approach that integrates patient-centred counselling and engagement of an adherence supporter for pregnant and breastfeeding women initiating HIV treatment (ie, antiretroviral therapy (ART)) or biomedical HIV prevention (ie, pre-exposure prophylaxis (PrEP)).

Methods Tonse Pamodzi 2 is a pilot study designed to provide acceptability, fidelity and clinical outcomes data on a set of behavioural interventions for adherence support. The study comprises two parallel randomised trials, enrolling HIV-positive pregnant women initiating ART (Trial 1, $n=100$ ) and HIV-negative pregnant women with risk of HIV acquisition and willing to initiate PrEP (Trial 2, $n=200$ ). Within each trial, participants are randomised 1:1 to either the intervention or control group. The Tonse Pamodzi adherence intervention comprises patient-centred counselling (adapted Integrated Next Step Counseling(iNSC)) and external adherence support tailored to the clinical context (ie, for ART or PrEP). Participants randomly assigned to the control group receive standard counselling based on local HIV guidelines. Participants are followed for 6 months. To assess intervention acceptability, we will employ a mixed method approach to describe participant engagement, satisfaction, and discussion content. We will audit and score recorded counselling sessions to evaluate the implementation fidelity of iNSC sessions. We will also assess clinical outcomes at 3 and 6 months for both Trial 1 (retention in care and viral suppression of HIV) and Trial 2 (retention in care, and plasma and intracellular tenofovir drug concentrations). Ethics and dissemination The study protocol was approved by the Malawi National Health Science Research Committee (19/05/2334) and the University of North
Strengths and limitations of this study

- We designed a status-neutral adherence intervention for pregnant and breastfeeding women that can be tailored to support adherence to either antiretroviral therapy for HIV-positive women or HIV preexposure prophylaxis for HIV-negative women at elevated risk of acquiring HIV.

- Our study is designed to evaluate the intervention's acceptability, fidelity and associated clinical outcomes.

- Study limitations include a relatively short follow-up period (ie, 6 months) and a targeted patient population; however, these features are consistent with other feasibility studies.

- Results from these pilot trials will inform the design of a larger study to assess the efficacy of the Tonse Pamodzi adherence intervention.

Carolina at Chapel Hill Institutional Review Board (191060).

Trial registration number NCT04330989.

\section{INTRODUCTION}

Over the past two decades, services for prevention of mother-to-child transmission (PMTCT) of HIV have expanded rapidly in sub-Saharan Africa, resulting in dramatic reductions in paediatric HIV. ${ }^{1-3}$ The progression of scientific, programmatic and policy advances have led to calls to eliminate motherto-child transmission of HIV (EMTCT). ${ }^{4-6}$ To achieve these ambitious benchmarks, 'statusneutral' approaches that offer universal services regardless of maternal HIV status are urgently needed. ${ }^{7}$ Such an approach can 
increase acceptability and reduce the stigma associated with HIV treatment and prevention services for pregnant and breastfeeding women.

In most African settings, PMTCT programmes have focused on the diagnosis, treatment, and retention of HIV-positive women within antenatal settings. The WHO and country-level policies support the provision of lifelong antiretroviral therapy (ART) for all HIV-positive pregnant and breastfeeding women (ie, the Option $\mathrm{B}+$ strategy), which may reduce HIV transmission rates to less than 2\%. ${ }^{8-11}$ Nevertheless, challenges remain. ART uptake among pregnant and breastfeeding women is not universal and nearly $20 \%$ of women drop out of care in the first 6 months on treatment. ${ }^{9-14}$ Further, poor adherence to ART can lead to antiretroviral drug resistance, treatment failure, and horizontal and vertical transmission. ${ }^{15-18}$

Incident maternal HIV infections-acquired during pregnancy and breast feeding-further contribute to the paediatric HIV burden, ${ }^{18}$ with up to $45 \%$ of new paediatric HIV cases attributable to new maternal HIV infection during pregnancy and breastfeeding in countries like Malawi. ${ }^{19}$ In 2018, the Joint United Nations Programme on HIV/AIDS estimated as many as 130000 women acquired HIV during pregnancy and breastfeeding, ${ }^{1}$ consistent with the high HIV incidence observed in clinical studies. ${ }^{20}{ }^{21}$ For most pregnant women who initially test HIV negative, few HIV prevention services are available, presenting a missed opportunity as these women are already engaged in care. Oral pre-exposure prophylaxis (PrEP) is recommended by the WHO to reduce horizontal HIV transmission and is considered safe and effective during pregnancy. ${ }^{22-24}$ Although the acceptability and feasibility of PrEP in antenatal/postnatal settings are encouraging, ${ }^{25-27}$ challenges to sustain uptake and adherence persist due to low risk of perception, perceived stigma and concerns about medication side effects. ${ }^{28}$
To reach the ambitious goals of EMTCT, effective and yet scalable approaches are needed to enhance current HIV services, including combination strategies that integrate proven biomedical and behavioural interventions. Given the critical role of medication adherence for both HIV treatment (ie, ART) and prevention (ie, PrEP), approaches that are adaptable and capable of being tailored to support antiretroviral use regardless of the context in which they are prescribed-treatment or prevention-may offer particular benefits in busy clinical settings. Such statusneutral interventions could broaden HIV services in maternal and child health settings. We developed an intervention combining patient-centred counselling (Integrated Next Step Counseling (iNSC)) and the inclusion of a participant-identified adherence supporter to enhance antiretroviral drug use during pregnancy and breastfeeding. In this protocol paper, we describe a pilot study to obtain data about the intervention's acceptability, fidelity and clinical outcomes.

\section{METHODS AND ANALYSIS \\ Study overview}

Tonse Pamodzi 2 (TP2) is a pair of pilot studies designed to evaluate an adherence support intervention for ART and PrEP. The term tonse pamodzi means 'all of us together' in Chichewa and Nyanja, signifying the importance of integrated facility-based and community-based adherence support. TP2 comprises two parallel randomised trials, evaluating an adapted integrated adherence support strategy for a different antenatal population. Trial 1 is enrolling HIV-positive pregnant women who have initiated ART; Trial 2 is enrolling HIV-negative pregnant women who meet local eligibility criteria to start PrEP (figure 1). The study enrolment began in March 2020 for Trial 1 and in June 2020 for Trial 2. The protocol is

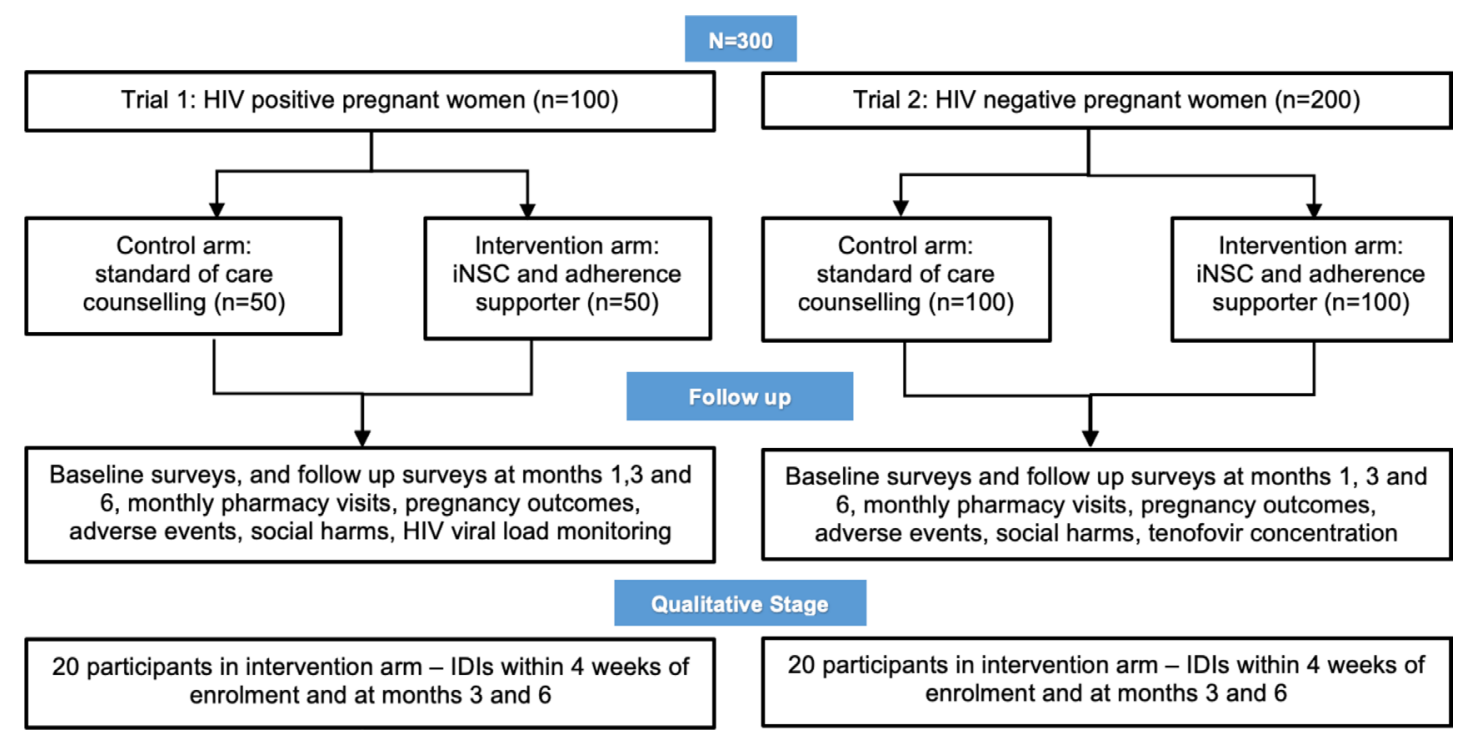

Figure 1 Consolidated Standards of Reporting Trials flow diagram. IDI, in-depth interviews; iNSC, Integrated Next Step Counseling. 


\section{i⿱宀 \\ Counseling

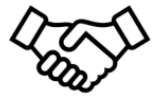 \\ Omukhulupilira \\ Adherence Support}

Integrated Next Step
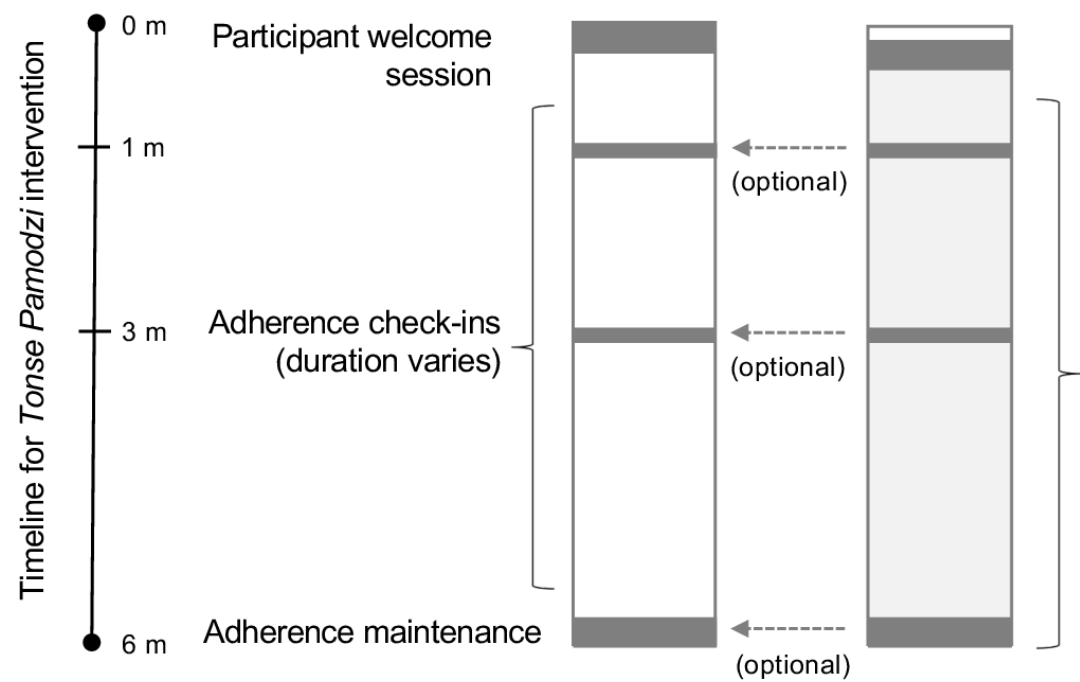

Omukhulupilira selection \& orientation

Ongoing adherence support and accompanied visits

Figure 2 Overview of the Tonse Pamodzi 2 adherence intervention.

registered in ClinicalTrials.gov. Online supplemental appendix 1 gives as a summary of the trial registration details.

\section{Study site}

The Bwaila District Hospital is a district level public hospital serving approximately one million people in Lilongwe and surrounding rural villages. It is the busiest maternity hospital in Malawi, with approximately 150 pregnant women attending antenatal care every day and over 1200 deliveries every month. Bwaila District Hospital has been providing PMTCT services since April 2002. Similar to many parts of Lilongwe, the antenatal HIV prevalence is estimated as $12 \% .^{29}$

\section{Adherence support: intervention group}

The TP adherence intervention was developed following intensive formative research-including systematic reviews; $;{ }^{21}{ }^{30}$ qualitative interviews with patients, healthcare workers and policymakers; ${ }^{26}{ }^{31}$ and mathematical modeling ${ }^{32}$-and expert consultation. It comprises two parts: an adaptation of iNSC and the optional inclusion of an adherence supporter who is from the patient's social network. Figure 2 provides an overview of the intervention session activities; a detailed description is included in online supplemental appendices 2 and 3.

A core component of our adherence support intervention is iNSC, a patient-centred counselling approach that adopts a systematic process or flow of conversation to promote patient engagement and autonomy as counsellors guide the conversation towards the identification of needs related to health and well-being, sexual health protection, and/or adherence (table 1).
iNSC has been used in several studies and programmes to support adherence for PrEP in different populations, but has never been tested among pregnant women. ${ }^{24} 3334$ The goal of iNSC is to foster a collaborative problemsolving environment that allows the participant to identify their individual needs to create, enhance or sustain overall well-being, sexual health through non-biomedical approaches, and adherence to antiretroviral drugs. iNSC frames the counselling sessions as a non-judgmental discussion to explore one's current experiences; one's vision for an alternative experience that would make health promotion easier/easiest to manage; and the facilitators, challenges and needs for progressing towards that improved situation. Although the content of these discussions is entirely tailored to the specific participants' situation, clinical context and priorities, the process of the conversation is identical across participants. Participants in the intervention arm receive iNSC at study enrolment and their visit at months 1,3 and 6 .

All individuals receiving the intervention are also invited to identify an adherence supporter or Omukhulupilira ('close confidant' in Chichewa and Nyanja) from their own social network. As part of the approach, this individual is trained to support the participant's use of ART or PrEP and may accompany the participant to study visits. The Omukhulupilira may be a partner, family member or friend chosen by the participant who can provide emotional, instrumental and informational social support. Selecting an Omukhulupilira is recommended, but not mandatory. Once nominated, an Omukhulupilira receives a brief in-person orientation training on how to provide positive support to the participant. This includes 


\begin{tabular}{|c|c|}
\hline Step & Content \\
\hline Introduce & Explain what you want to discuss, why and ask permission \\
\hline Frame discussion & $\begin{array}{l}\text { Frame discussion to two components, first about general well-being and then about adherence. Steps } \\
\text { below will be repeated for each component. }\end{array}$ \\
\hline Review & Check in on previous goals/discussions, close and move into current experiences (follow-up visits only) \\
\hline Explore & Discuss socioecological factors that challenge or could optimise a specific behaviour \\
\hline Tailor & Reflect on context and experiences shared to tailor remainder of the discussion \\
\hline Identify & $\begin{array}{l}\text { Ask what would be needed to happen for the situation (identified above) to be slightly better, easier to } \\
\text { handle or be more manageable }\end{array}$ \\
\hline Strategise* & Ask how the participant might consider addressing this need \\
\hline Agree* & Ask the participant if she would agree to try out one or more strategies to address the identified need \\
\hline Transition/close* & Move to a new topic and repeat the flow or close the discussion \\
\hline
\end{tabular}

${ }^{*}$ These steps may be repeated in a joint discussion with the adherence supporter (ie, Omukhulupilira), if he/she is present and the participant agrees.

basic HIV knowledge, the importance of antiretroviral adherence, potential drug side effects, and practical strategies for supporting adherence. This intervention component is based on the premise that social support provides access to essential information and resources to encourage specific health behaviours. ${ }^{35}$

\section{Adherence support: current standard of care}

Participants randomly assigned to the control group receive standard counselling in PMTCT, safe obstetrics and newborn care, based on the Malawi Ministry of Health guidelines. ${ }^{36}$ In addition, all participants receive education regarding HIV/AIDS-including HIV treatment and prevention-when they initiate either ART or PrEP. The content of this education comprises the basics of HIV transmission and prevention, stages of HIV infection, the role of ART or PrEP for PMTCT and their mechanisms of action, dosing and adherence guidance, and drug safety and side effects.

\section{Recruitment and retention procedures}

Participants are recruited from the antenatal clinic at Bwaila District Hospital in Lilongwe, Malawi. Study staff provide educational talks about the study in the antenatal waiting areas. Pregnant women interested in the study are booked for screening at the research clinic and undergo informed consent before study procedures begin. Once participants are enrolled in the trial, the study team uses a multipronged strategy-including telephone contact, community outreach and peer engagement-to retain those in both arms to minimise losses to follow-up. The study team closely monitors retention and addresses any issues prospectively.

\section{Randomisation}

Participants are randomised separately within each trial and the randomisation procedures are similar between the two parallel studies. Within each trial, participants are randomly assigned 1:1 to either the intervention or control arm using a permuted block randomisation design. ${ }^{37}$ The study statistician (KRM) independently generated the randomisation assignments using SAS V.9.4 software. Opaque, sealed randomisation envelopes for Trial 1 and Trial 2 were numbered in advance of enrolment by an independent data staff member and stored in sequential order in separate boxes. Team members preparing these envelopes are not involved in ascertaining eligibility or assessing outcomes. Once eligibility is confirmed and the participant provides informed consent (online supplemental appendix 4), the research nurse obtains the next sequential envelope from the box for the randomisation assignment, enters the randomisation identification number on the envelope into the participant's linkages form and then opens the envelope to determine randomisation assignment. Due to the nature of the intervention and data collection instruments, it is not possible to blind clinic staff, study statisticians or participants on the assigned randomisation arm. However, the laboratory technicians responsible for measuring biological endpoints are blinded to the randomisation assignment.

\section{Considerations for Trial 1 (ART adherence support)}

Eligibility criteria for Trial 1: We are enrolling HIVpositive women 18 years of age or older with documented pregnancy by urine pregnancy test or physical examination. Other eligibility criteria include initiation on firstline ART within the past 30 days, for the first time or after treatment interruption of 6 months or longer (if previously started, but stopped ART). HIV-positive women initiating ART may be enrolled on the same day if they meet the eligibility criteria. The women should be willing to provide written informed consent, remain in the study site's catchment area throughout study follow-up, and comply with the visit schedule. Women with risk for intimate partner violence (IPV) or social harms resulting from participation as assessed by study personnel are excluded. 
Table 2 Schedule of evaluations for Trial 1-ART adherence support for pregnant women living with HIV

Clinic visits

Adherence

Enrolment

Month

\section{Month $3 \quad$ Month 6} assessment

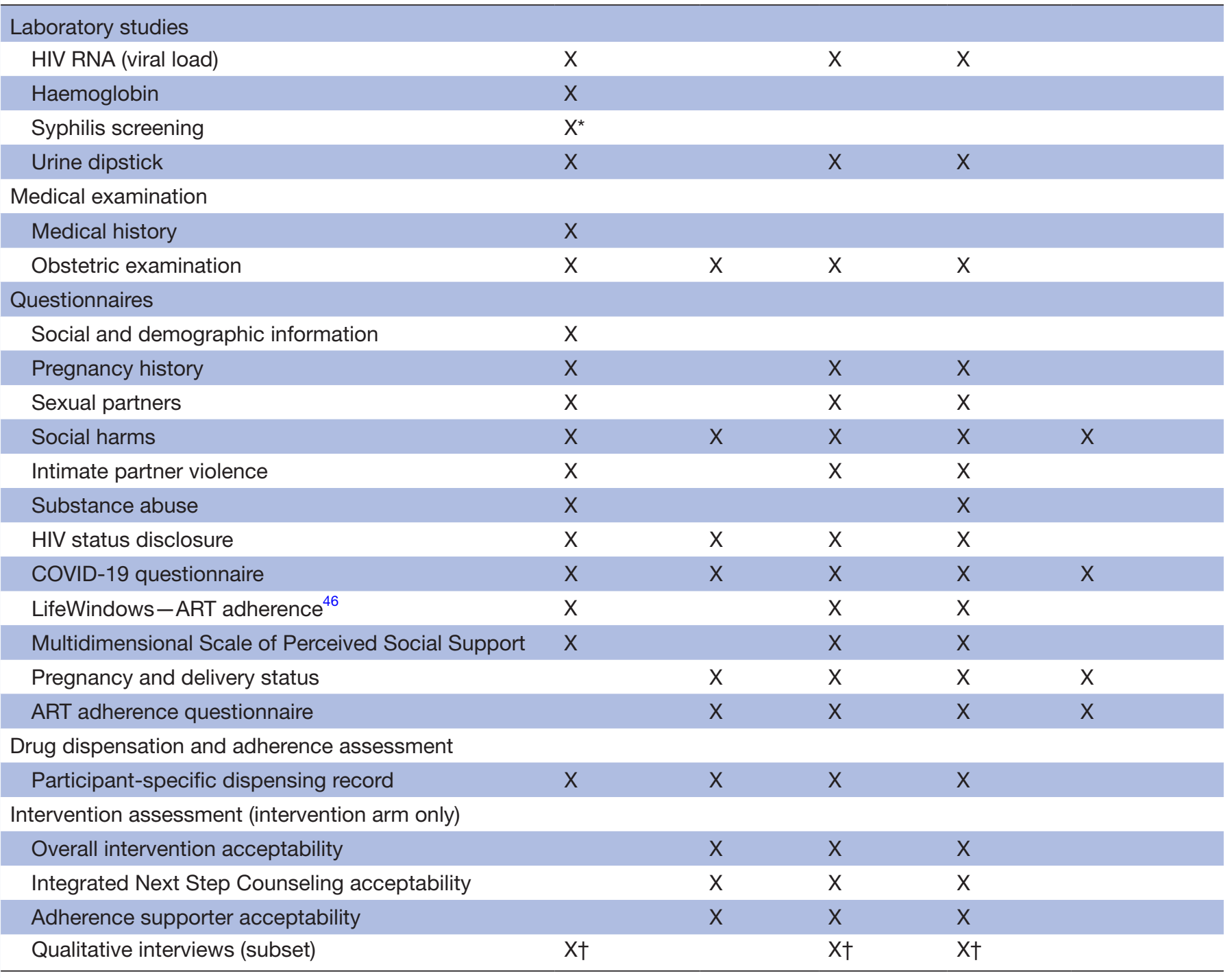

*Will perform test if no results available in the medical record.

†Schedule additional procedures within 30 days of visit for subset of participants enrolled in this component.

ART, antiretroviral therapy.

Study activities for Trial 1: Visits occur at enrolment, and at months 1,3 and 6 (table 2 ). The study procedures at these scheduled visits include collecting blood and urine specimens for the following laboratory tests: HIV RNA (viral load), haemoglobin, syphilis screening (if not available in the antenatal record) and urine dipstick (table 2). These specimens are collected for safety monitoring and outcome measurement. Urine dipstick is collected per local standard of care. HIV viral load is measured onsite using the HIV RNA PCR on M2000 platform (Abbott Laboratories, Lake Forest, Illinois, USA) at the University of North Carolina Project Malawi Central Laboratory (Lilongwe, Malawi). Most index pregnancies are expected to end prior to the participant's 6-month study visit, and we collect obstetric and neonatal information when the pregnancy outcome is available. In the event that the index pregnancy is ongoing at the participant's 6-month study visit, we schedule additional monthly follow-up visits until the participant can report an obstetrical outcome. ART regimens are prescribed according to local HIV guidelines: ${ }^{36}$ tenofovir disoproxil fumarate (TDF) and lamivudine with either dolutegravir or efavirenz. The dispensation of ART is coordinated with the local PMTCT/ART programme at the study facility. We collect information about prescribed ART drugs, pharmacy dispensations and adherence evaluations at short visits every month, timed with routine clinic visits. 


\section{Considerations for Trial 2 (PreP adherence support)}

Eligibility criteria for enrolment in Trial 2: In Trial 2, we enrol HIV-negative pregnant women 18 years of age or older willing to initiate and continue daily oral PrEP, coformulated as TDF and emtricitabine (TDF/FTC; 200/300 mg). To be eligible, candidates must report one or more of the following risk factors for HIV acquisition in the past 12 months: known positive or unknown partner HIV status, multiple sexual partners, diagnosis of a sexually transmitted infection, use of postexposure prophylaxis, use of shared injection material or equipment, or an unspecified concern about HIV acquisition. Other eligibility criteria include documented pregnancy by urine pregnancy test or physical examination, a documented negative HIV status within the past 3 months, plans to remain in the study site's catchment area throughout study follow-up, willingness to provide informed consent and comply with the visit schedule, and interest in initiating and continuing PrEP throughout study follow-up. We exclude women who test positive for hepatitis B surface antigen at the time of screening, have renal insufficiency (defined as creatinine clearance $<90 \mathrm{~mL} / \mathrm{min}$ ), have a history of known renal parenchymal disease or have a known single kidney at the time of screening. Women with risk for IPV or social harms resulting from participation as assessed by study personnel, are excluded.

Activities for Trial 2: In Trial 2, participants undergo screening procedures to confirm their HIV and hepatitis B status, both of which must be negative before enrolment and randomisation. Study visits will occur at enrolment and at months 1, 3 and 6 (table 3 ). We use oral PrEP coformulated as TDF/FTC, to be dosed on a oncedaily basis. The packaged product is dispensed for Trial 2 participants by onsite study staff. Participants begin daily administration of TDF/FTC on the day of randomisation and this will continue through month 6 . PrEP is dispensed for 1-3 months at a time, depending on local control measures for COVID-19 (see COVID-19 considerations below). Study laboratory tests at baseline include rapid HIV antibody screening, alanine transaminase, creatinine, syphilis screening (if not available in the antenatal record) and urine dipstick. At follow-up visits, tests include HIV antibody screening, urine dipstick, creatinine, tenofovir (TFV) concentrations, and intracellular TFV-diphosphate (TFVdp) measurements. These specimens are collected both for routine safety monitoring for PrEP and as outcome measurements. As in Trial 1, if the index pregnancy is ongoing at the 6-month study visit, we will schedule monthly follow-up visits until the participant reports a pregnancy outcome. Specimens for TFV concentration and TFVdp measurements are stored at the local site laboratory and then shipped for processing at the University of North Carolina at Chapel Hill by the Clinical Pharmacology and Analytical Chemistry Core of the Center for AIDS Research.

For women who test HIV positive during the study, TDF/FTC will be discontinued while the diagnosis is confirmed. We will also collect a specimen for HIV RNA (ie, viral load) and storage for HIV resistance testing. If the HIV diagnosis is confirmed, the participant completes exit study procedures (ie, analogous to the 6-month visit) and receives a guided referral to the HIV care and treatment clinic at the study site.

\section{Acceptability and fidelity endpoints}

Mixed methods assessment of intervention acceptability: Among those randomised to the intervention group, we assess the acceptability of the integrated intervention and its component parts (ie, adherence supporter and iNSC) at each study visit. This includes questions about overall satisfaction and, if the intervention were to be made available again, likelihood of continued use. We inquire about aspects of the iNSC experience, including the discussion content, counsellor engagement and perceived effect on adherence behaviours. We also ask about whether they are working with an Omukhulupilira and, if so, his/her level of engagement in adherence support and perceived effect on adherence behaviours. Questions are structured with binary outcomes (eg, yes/no) or using a five-point Likert scale. To complement these quantitative assessments, individual semi-structured interviews (SSIs) are conducted to further explore participant experiences with the TP intervention. We are interested in individual-level engagement (including satisfaction with the different components), as well as barriers and facilitators to participation. With three interviews planned (ie, following enrolment, and at 3-month and 6-month visits), our approach provides a longitudinal assessment of the intervention. We are recruiting a subset of 40 study participants, all from the intervention group, divided equally between the two trials.

Quantitative assessment of iNSC fidelity: We conduct detailed audits of iNSC sessions for all participants assigned to the intervention arm from both trials $(n=150$ total). A case report form is used to document fidelity to each component of the iNSC session. Two reviewers independently rate the iNSC session according to key domains, providing both objective and subjective measures about the quality of the counselling. Through this process, our audit staff also assess the appropriateness of documentation in the primary case report forms, including misinformation and missing data. This exercise provides critical feedback loops to improve counselling over time.

\section{Clinical endpoints}

In addition, we collect longitudinal participant outcomes data at 3 and 6 months for both trials. In Trial 1 (HIVpositive women), we will examine an endpoint of retention in care with viral suppression of HIV $(<40$ copies/ $\mathrm{mL}$ ). In Trial 2 (HIV-negative women), we will examine retention in care with adherence to PrEP as measured by plasma and intracellular TFV drug concentrations at 6 months following study enrolment. Using published algorithms,${ }^{38}$ results from the plasma and upper layer of packed cells assays are combined to develop a composite adherence score (table 4). Our Trial 2 clinical outcome is 
Table 3 Schedule of evaluations for Trial 2-PrEP adherence support for HIV-negative pregnant women

\section{Clinical visits}

\section{Screening Enrolment Month 1 Month 3 Month 6 Monthly}

Adherence assessment

HIV confirmatory testing

(if rapid HIV test is positive)

\begin{tabular}{|c|c|c|c|c|c|c|c|}
\hline \multicolumn{8}{|l|}{ Laboratory studies } \\
\hline Rapid HIV antibody test & $x$ & $x$ & & $x$ & $x$ & & \\
\hline Hepatitis B antigen & $x$ & & & & & & \\
\hline Alanine aminotransferase & & $x$ & & & & & \\
\hline Creatinine & $x$ & & & $x$ & $x$ & & \\
\hline Syphilis screening & & & & & & & \\
\hline Urine dipstick & & $x$ & $x$ & $x$ & $x$ & & \\
\hline Tenofovir concentration & & & & $x$ & $x$ & & \\
\hline HIV RNA (viral load) & & & & & & & $x$ \\
\hline $\begin{array}{l}\text { Storage for HIV resistance } \\
\text { testing }\end{array}$ & & & & & & & $x$ \\
\hline \multicolumn{8}{|l|}{ Medical examination } \\
\hline Medical history & $x$ & & & & & & \\
\hline Obstetric examination & $x$ & $x$ & $x$ & $x$ & $x$ & & \\
\hline \multicolumn{8}{|l|}{ Questionnaires } \\
\hline $\begin{array}{l}\text { Social and demographic } \\
\text { information }\end{array}$ & & $x$ & & & & & \\
\hline Pregnancy history & & $x$ & & $x$ & $x$ & & \\
\hline Sexual partners & & $x$ & & $x$ & $x$ & & \\
\hline COVID-19 questionnaire & & $x$ & $x$ & $x$ & $x$ & $x$ & \\
\hline Social harms & & $x$ & $x$ & $x$ & $x$ & $x$ & \\
\hline Intimate partner violence & & $x$ & & $x$ & $x$ & & \\
\hline Substance abuse & & $x$ & & & $x$ & & \\
\hline $\begin{array}{l}\text { Motivating factors for PrEP } \\
\text { use }\end{array}$ & & $x$ & & & & & \\
\hline $\begin{array}{l}\text { Perceived HIV risk } \\
\text { assessment }\end{array}$ & & $x$ & & & $x$ & & \\
\hline PrEP use disclosure & & $x$ & $x$ & $x$ & $x$ & & \\
\hline $\begin{array}{l}\text { LifeWindows-PrEP } \\
\text { adherence }^{46}\end{array}$ & & $x$ & & $x$ & $\mathrm{x}$ & & \\
\hline $\begin{array}{l}\text { Multidimensional Scale of } \\
\text { Perceived Social Support }\end{array}$ & & $x$ & & & $x$ & & \\
\hline $\begin{array}{l}\text { PrEP adherence } \\
\text { questionnaire }\end{array}$ & & & $x$ & $x$ & $x$ & $x$ & \\
\hline $\begin{array}{l}\text { Pregnancy and delivery } \\
\text { status }\end{array}$ & & & $\mathrm{x}$ & $x$ & $\mathrm{x}$ & $\mathrm{x}$ & \\
\hline \multicolumn{8}{|c|}{ Drug dispensation and adherence assessment } \\
\hline $\begin{array}{l}\text { Participant-specific } \\
\text { dispensing record }\end{array}$ & & $\mathrm{x}$ & $\mathrm{x}$ & $x$ & $\mathrm{x}$ & $\mathrm{x}$ & \\
\hline \multicolumn{8}{|c|}{ Intervention assessment (intervention arm only) } \\
\hline $\begin{array}{l}\text { Overall intervention } \\
\text { acceptability }\end{array}$ & & & $x$ & $x$ & $\mathrm{x}$ & & \\
\hline $\begin{array}{l}\text { Integrated Next Step } \\
\text { Counseling acceptability }\end{array}$ & & & $\mathrm{x}$ & $\mathrm{x}$ & $\mathrm{x}$ & & \\
\hline
\end{tabular}

Continued 


\section{Clinical visits}

Adherence assessment

HIV confirmatory testing

(if rapid HIV test is positive)

\section{Screening Enrolment Month 1 Month 3 Month 6 Monthly}

Adherence supporter acceptability

Qualitative interviews

$\mathrm{X} \dagger$

$\mathrm{X} \dagger$

$\mathrm{X} \dagger$

*Will perform test if no results are available in the medical record.

†Schedule additional procedures within 30 days of visit for subset of participants enrolled in this component.

PrEP, pre-exposure prophylaxis.

based on functional adherence, defined as 4-5 or more doses/week (scores of 4 or 5 ).

In line with pilot designs, our study is not formally powered to detect clinically meaningful differences between study arms (see the Sample size section). Instead, these data will be used to assess the feasibility of future trials, including in the areas of recruitment and retention. These preliminary endpoint data will also provide baseline estimates for future studies, especially for PrEP use among pregnant and breastfeeding women, where adherence data remain scarce.

\section{Safety monitoring}

At each study visit, the study staff will evaluate participants for social harms, IPV and AEs (Adverse Events). We will use standardised instruments to screen for social harms and IPV. Social harms involve problems with other people as a result of study participation. IPV involves physical or sexual violence experienced with a partner. An AE will be defined as any untoward medical occurrence in a study participant, including an abnormal physical examination or laboratory finding, symptom or disease, temporally associated with the individual's participation in the research, whether or not considered related to participation in the research. The severity of events will be graded using the National Institute of Health's Division of AIDS Table for Grading the Severity of Adult and Pediatric Adverse Events. Participant deaths will be recorded along with the contributing cause(s) of death. We will also record information on all serious adverse events occurring in participants, whether or not they are related to study participation or the study drug. Because our combination intervention is designed to enhance adherence-and because the Malawi Ministry of Health already recommends the prescribed antiretroviral regimens-a data monitoring committee has not been convened.

\section{Sample size}

In Trial 1, we plan to enrol 100 HIV-positive pregnant women, randomised 1:1 between the intervention and control arms ( $\mathrm{n}=50$ each). In Trial 2, we plan to enrol 200 HIV-negative pregnant women, randomised 1:1 between the intervention and control arms $(\mathrm{n}=100$ each). Similar to other pilot studies, these sample sizes were selected based on practical considerations, including recruitment ability, participant flow, and budgetary constraints. We enrolled larger number of HIV-negative women in Trial 2 because assessments of PrEP adherence during pregnancy and breastfeeding are limited in the medical literature. These sample sizes were deemed sufficient to evaluate the acceptability and fidelity endpoints described above. We also conducted exploratory precision and power calculations for our clinical endpoints over a range of potentially meaningful effect sizes.

\section{Data management}

Clinical and behavioural data collected during this study are collected on case report forms and through REDCap

\begin{tabular}{llll}
\hline Table 4 & Adherence composite scores based on TFV and TFVdp concentrations, with doses estimated/interval \\
\hline Score & TFV in plasma & TFVdp in ULPC & Estimates doses per interval \\
\hline 0 & None detectable & $<10000 \mathrm{fmol} / \mathrm{mL}$ & Low number or no doses in the interval \\
1 & Detectable & $<10000 \mathrm{fmol} / \mathrm{mL}$ & A few doses in the entire interval \\
2 & Any level & $10000-100000 \mathrm{fmol} / \mathrm{mL}$ & $1-2$ doses $/$ week \\
3 & $<10 \mathrm{ng} / \mathrm{mL}$ & $>100000 \mathrm{fmol} / \mathrm{mL}$ & $\begin{array}{l}\text { Several doses early in the interval, followed by a stop in the } \\
1-2 \text { weeks leading up to sampling visit }\end{array}$ \\
4 & $10 \mathrm{ng} / \mathrm{mL}$ or higher & $100000-1000 \mathrm{000} \mathrm{fmol} / \mathrm{mL}$ & $4-5$ doses/week \\
5 & $10 \mathrm{ng} / \mathrm{mL}$ or higher & $>1000000 \mathrm{fmol} / \mathrm{mL}$ & Approximately daily dosing
\end{tabular}

Adapted from Corneli et al. ${ }^{38}$

TFV, tenofovir; TFVdp, tenofovir diphosphate; ULPC, upper layer packed cells. 
software. ${ }^{39}$ Access to study data is restricted to a limited number of team members (data coordinator, study coordinator and study investigator). For the qualitative interviews, the SSI audio recordings are transcribed and translated for analysis. All identifiers are redacted from the interview transcripts before analysis. Weekly data monitoring reports, including the performance indicators, are generated to monitor study progress. Deidentified data will be made available via existing online data repositories once the planned analyses are completed and data sharing agreements are established with proposed investigators and institutions.

\section{Confidentiality}

Measures are being taken to ensure the safety of data and confidentiality of all our study participants. All participants are assigned a unique coded study identification numbers (ID) number. The interview guides will not capture names of the participants, but only coded study ID. No participants will be identified in any report or publication about this study. Clinical information with individual identifiers will not be released without the written permission of the participant. We expect these procedures to protect participant confidentiality adequately.

\section{Data analysis}

In our assessment of intervention acceptability, we will tabulate participant responses about engagement, satisfaction and discussion content. Only participants in the intervention group will be included in these analyses and the results will be stratified by trial. Outcomes will be reported as a proportion, with precision quantified using 95\% CIs. We will examine the responses to our five-point Likert scale and, depending on their distribution, report them as either categorical or binary variables. To complement these qualitative data, we will analyse SSI data to gain additional insights about intervention acceptability. Data will be analysed using established techniques that include coding, memoing and matrices to summarise and interpret key patterns in the data. Comparative and thematic analyses will be used to provide an in-depth understanding of the experiences related to HIV testing.

To assess fidelity, each iNSC session will be scored (range 0-100) and then stratified by individual participant, type of study visit, staff member providing iNSC and calendar time. This will allow us to describe trends in fidelity over the course of participant follow-up time and calendar time. We will describe scores overall and by steps within iNSC.

Analyses of clinical endpoints will be conducted separately for each trial. Given the nature of these pilot studies, emphasis will be placed on estimation and precision. In Trial 1, we will assess ART adherence by evaluating the viral suppression status of each study participant at 3 and 6 months after randomisation. Participants not lost to follow-up will be classified as either virally suppressed ( $<40$ copies $/ \mathrm{mL}$ ) or not virally suppressed ( $\geq 40$ copies/ $\mathrm{mL}$ ). The proportion of women retained in care with viral suppression will be compared between randomisation arms by estimating the risk difference and corresponding 95\% CI. In Trial 2, we will assess PrEP adherence by evaluating retention in care and functional PrEP use for each study participant at 3 and 6 months. Mirroring our approach in Trial 1, the proportion of women retained in care with functional PrEP use will be compared between randomisation arms using a risk difference and corresponding $95 \%$ CI. Women who do not attend the month 6 visit will be counted as failures and contribute to the analysis denominator.

The incidence of social harms, IPV and other AEs will be estimated by randomisation arm and, where possible, by individual event types. Study retention will be described for each scheduled visit using frequency tables, and reasons for attrition will be described. Adherence to ART and PrEP will also be measured both by self-report and pharmacy measures. A medication possession ratio will be estimated and described using summary statistics, using established thresholds to dichotomise this endpoint. ${ }^{40}$ We will compare these composite adherence outcomes between randomisation arms using a risk difference and corresponding $95 \%$ CI.

\section{Patient and public involvement}

Prior to commencing recruitment, the local Community Advisory Board (CAB) was engaged to review the study aims and recruitment procedures. The CAB consists of local community and religious leaders, community representatives and adolescents representing the youth. CAB meetings were held bimonthly to review progress in enrolment and retention, and to solicit feedback about ongoing successes and challenges with study implementation.

\section{COVID-19 considerations}

Due to the ongoing COVID-19 pandemic, ${ }^{42}$ we have modified our activities to increase the safety of staff and participants. All study personnel practice personal safety measures when interacting with others, including personal protective equipment, frequent handwashing and physical distancing. ${ }^{43}$ All participants wear face masks when being attended to on the study site. To minimise the number of clinic visits, we dispense study drugs (either ART or PrEP) for longer durations-aligned with study activities-and conduct monthly adherence assessments via phone. All directives from the Malawi Ministry of Health are followed and an information sheet is used to provide participants with up-to-date information on COVID-19 preventive measures.

\section{Ethics and dissemination}

The study protocol has been reviewed and approved by the Malawi National Health Science Research Committee $(19 / 05 / 2334)$ and the University of North Carolina at Chapel Hill Institutional Review Board (19-1060). Any protocol modifications are submitted to and approved by these committees before any change in implementation. 
Study findings will be disseminated through appropriate local channels, including academic and public health research symposia.

\section{DISCUSSION}

The TP2 study comprises two parallel trials to support antiretroviral adherence in the setting of antenatal and postnatal care, both for ART (Trial 1) and PrEP (Trial 2 ). This pilot study will provide important data on intervention acceptability, fidelity and clinical outcomes. Our overarching goal is to assess these attributes, while evaluating the feasibility of the TP2 adherence intervention for future efficacy trials.

Our trial focuses on two different pregnant and breastfeeding populations that may benefit from adherence interventions-HIV-positive women on ART and HIV-negative women on PrEP. For the HIV-positive group, we focused on those who had recently initiated HIV treatment or restarted following a prolonged (ie, greater than 6 months) interruption. We reasoned that the timing of our combination behavioural intervention may be most relevant during this start/restart window. However, we also recognise the importance of adherence support for those on long-term ART, even in shorter periods. If shown to be acceptable in our pilot study, we would look to expand the eligibility criteria in follow-on studies.

Similar to other settings in sub-Saharan Africa, the provision of PrEP during pregnancy and breastfeeding in Malawi remains limited. This study will provide important acceptability data about PrEP use, including self-reported and objective measures of adherence. Although we considered common challenges to PrEP delivery in the design of our study, some barriers were difficult to address. For example, the integration of PrEP dispensation with routine antenatal/postnatal visits may promote adherence and persistence. ${ }^{274}$ However, such evaluation schedules did not align with our adherence assessments or our relatively short (ie, 6month) follow-up period. Nevertheless, as part of our acceptability assessments, we will gather data that can inform future PrEP delivery strategies.

While we expect to gain insights into antiretroviral adherence during pregnancy and breastfeeding, the design of this pilot study emphasises the former period. All participants are enrolled during pregnancy, with the expectation that most will deliver prior to the 6 months and continue in follow-up. The vast majority of new mothers in Malawi opt to breastfeed their children ${ }^{45}$ and, as such, we did not make the intention to breastfeed a separate eligibility criterion for the trial. Our assessments of acceptability and fidelity will consider a women's pregnancy status (ie, antenatal or postnatal) and allow us to evaluate the intervention across these related, but distinctly different time periods. The study will also provide some descriptive data about PrEP safety in pregnancy and breastfeeding, both for the participant and her newborn.

In summary, the TP2 study evaluates a new combination intervention to support antiretroviral adherence. This pilot study will provide important data about the intervention's acceptability, fidelity and clinical outcomes promoting consistent ART and PrEP use. Once completed, our study will provide insight into broad strategies for HIV prevention and treatment that are status neutral, and able to support both HIV-positive and HIV-negative pregnant women in sub-Saharan Africa.

\section{Author affiliations}

${ }^{1}$ University of North Carolina Project-Malawi, Lilongwe, Malawi

${ }^{2}$ Department of Health Policy and Systems, University of Zambia School of Medicine, Lusaka, Zambia

${ }^{3}$ Department of Obstetrics and Gynecology, School of Medicine, University of North Carolina at Chapel Hill School of Medicine, Chapel Hill, North Carolina, USA

${ }^{4}$ Department of Health Behavior, University of North Carolina at Chapel Hill Gillings School of Global Public Health, Chapel Hill, North Carolina, USA

${ }^{5}$ Department of Epidemiology, University of North Carolina at Chapel Hill Gillings

School of Global Public Health, Chapel Hill, North Carolina, USA

${ }^{6}$ Department of Health Behavior and Health Education, University of Michigan

School of Public Health, Ann Arbor, Michigan, USA

${ }^{7}$ Center for AIDS Research, University of North Carolina at Chapel Hill, Chapel Hill, North Carolina, USA

${ }^{8}$ Lighthouse Trust, Lilongwe, Malawi

${ }^{9}$ Department of Medicine, School of Medicine, University of North Carolina, Chapel Hill, North Carolina, USA

${ }^{10}$ Department of Global Health, University of Washington, Seattle, Washington, USA

${ }^{11}$ School of Public Health and Family Medicine, College of Medicine University of

Malawi, Blantyre, Malawi

${ }^{12}$ Department of HIV and AIDS, Ministry of Health Malawi, Lilongwe, Malawi

${ }^{13}$ Institute for Social and Preventive Medicine (ISPM), University of Bern, Bern, Switzerland

\section{Twitter Friday Saidi @DrFridaySaidi}

Acknowledgements We acknowledge the research team members at University of North Carolina Project-Malawi for their commitment and preparatory work to see the study open to enrolment. We thank the Bwaila District Hospital management and staff for their support. We would like to also thank the management and staff at Lighthouse Trust for their support.

Contributors BHC and WM are the trial's protocol chairs. In Malawi, FS is the lead investigator, TP is the study coordinator, and BM is the data coordinator. BHC, WM, FS, KF, AMG, NER, SP, LAG, KM and LMH developed the study protocol. KRA, FS, BHC, KF, NER, SM, LMH and WM developed the intervention manual and KRA and KF developed the participatory workbook for Integrated Next Step Counseling. KF, SM and LMH developed the qualitative component. TK and SP advised the study team and contributed scientific expertise. FS, TP, LAG, $\mathrm{LMH}$ and BM oversaw data collection and data management activities. KRM, LAG, BHC and FS planned the analyses. BHC, WM, FS, KF, KRA and TP oversaw implementation of study procedures. All the authors have contributed to the development of this manuscript. They have read and approved the final version for publication.

Funding The US National Institute of Allergies and Infectious Diseases (NIAID, R01 Al131060) funds the Tonse Pamodzi 2 trial. Additional investigator, trainee and administrative support is provided by NIAID (K24Al120796, P30 Al050410), National Institute of Mental Health (K01 MH121186 and R00 MH104154) and Fogarty International Center (D43 TW009340, D43 TW010060).

Disclaimer Funders were not involved in the study design development, writing of the protocol and in the decision to submit this article for publication.

Competing interests None declared.

Patient consent for publication Not applicable

Provenance and peer review Not commissioned; externally peer-reviewed. 
Supplemental material This content has been supplied by the author(s). It has not been vetted by BMJ Publishing Group Limited (BMJ) and may not have been peer-reviewed. Any opinions or recommendations discussed are solely those of the author(s) and are not endorsed by BMJ. BMJ disclaims all liability and responsibility arising from any reliance placed on the content. Where the content includes any translated material, BMJ does not warrant the accuracy and reliability of the translations (including but not limited to local regulations, clinical guidelines, terminology, drug names and drug dosages), and is not responsible for any error and/or omissions arising from translation and adaptation or otherwise.

Open access This is an open access article distributed in accordance with the Creative Commons Attribution Non Commercial (CC BY-NC 4.0) license, which permits others to distribute, remix, adapt, build upon this work non-commercially, and license their derivative works on different terms, provided the original work is properly cited, appropriate credit is given, any changes made indicated, and the use is non-commercial. See: http://creativecommons.org/licenses/by-nc/4.0/.

\section{ORCID iDs}

Friday Saidi http://orcid.org/0000-0003-1190-1499

Wilbroad Mutale http://orcid.org/0000-0002-4891-6750

Benjamin H Chi http://orcid.org/0000-0002-1435-8455

\section{REFERENCES}

1 UNAIDS. Fast-Track - Ending the AIDS epidemic by 2030. Geneva, Switzerland: UNAIDS, 2014. https://www.unaids.org/en/resources/ documents/2014/JC2686_WAD2014report

2 Joint United Nations Programme on HIV/AIDS, editor. Countdown to zero: global plan towards the elimination of new HIV infections among children by 2015 and keeping their mothers alive, 2011-2015. Geneva, Switzerland: UNAIDS, 2011: 44.

3 Joint United Nations Programme on HIV/AIDS. Together we will end AIDS. Geneva. Switzerland: Joint United Nations Programme on HIV/ AIDS (UNAIDS), 2012

4 Chi BH, Adler MR, Bolu O, et al. Progress, challenges, and new opportunities for the prevention of mother-to-child transmission of HIV under the US president's emergency plan for AIDS relief. $J$ Acquir Immune Defic Syndr 2012;60 Suppl 3:S78-87.

$5 \mathrm{Chi} \mathrm{BH}$, Stringer JSA, Moodley D. Antiretroviral drug regimens to prevent mother-to-child transmission of HIV: a review of scientific, program, and policy advances for sub-Saharan Africa. Curr HIVIAIDS Rep 2013;10:124-33.

6 Joint United Nations Programme on HIV/AID. 2015 progress report on the global plan towards the elimination of new HIV infections among children and keeping their mothers alive. Geneva, Switzerland: UNAIDS, 2015. https://www.unaids.org/sites/default/ files/media_asset/JC2774_2015ProgressReport_GlobalPlan_en.pdf

7 Myers JE, Braunstein SL, Xia Q, et al. Redefining prevention and care: a Status-Neutral approach to HIV. Open Forum Infect Dis 2018:5:ofy097.

8 Schouten EJ, Jahn A, Chimbwandira F, et al. Is option B+ the best choice? The Lancet 2013;381:1272-3.

9 Kalua T, Tippett Barr BA, van Oosterhout JJ, et al. Lessons learned from option B+ in the evolution toward "Test and Start" from Malawi, Cameroon, and the United Republic of Tanzania. J Acquir Immune Defic Syndr 2017;75 Suppl 1:S43-50.

10 Tippett Barr BA, van Lettow M, van Oosterhout JJ, et al. National estimates and risk factors associated with early mother-to-child transmission of HIV after implementation of option B+: a crosssectional analysis. Lancet HIV 2018;5:e688-95.

11 Haberer JE, Sabin L, Amico KR, et al. Improving antiretroviral therapy adherence in resource-limited settings at scale: a discussion of interventions and recommendations. J Int AIDS Soc 2017;20:21371.

12 Rollins NC, Essajee SM, Bellare N, Doherty M, Hirnschall GO, et al. Improving retention in care among pregnant women and mothers living with HIV: lessons from INSPIRE and implications for future who guidance and monitoring. J Acquir Immune Defic Syndr 2017;75 Suppl 2:S111-4.

13 Chimbwandira F, Mhango E, Makombe S. Impact of an innovative approach to prevent mother-to-child transmission of HIV--Malawi, July 2011-September 2012. MMWR Morb Mortal Wkly Rep 2013;62:148-51.

14 Tenthani L, Haas AD, Tweya $\mathrm{H}$, et al. Retention in care under universal antiretroviral therapy for HIV-infected pregnant and breastfeeding women ('Option B+') in Malawi. AIDS 2014;28:589-98.

15 Nachega JB, Uthman OA, Anderson J, et al. Adherence to antiretroviral therapy during and after pregnancy in low-income, middle-income, and high-income countries. AIDS 2012;26:2039-52.
16 Kirsten I, Sewangi J, Kunz A, et al. Adherence to combination prophylaxis for prevention of mother-to-child-transmission of HIV in Tanzania. PLoS One 2011;6:e21020.

17 Haas AD, Msukwa MT, Egger M. Adherence to antiretroviral therapy during and after pregnancy: cohort study on women receiving care in Malawi's option B+ program. Clin Infect Dis 2016;63:1227-35.

18 UNAIDS. improving UNAIDS' paediatric and adolescent estimates. Geneva, Switzerland: UNAIDS, 2020. https://www.unaids.org/ sites/default/files/media_asset/improving-unaids-paediatric-andadolescent-estimates_en.pdf

19 UNICEF. Last-mile-to-emtct_whitepaper_unicef2020.Pdf, 2020. Available: http://www.childrenandaids.org/sites/default/files/2020-02/ Last-Mile-To-EMTCT_WhitePaper_UNICEF2020.pdf

20 Drake AL, Wagner A, Richardson $\bar{B}$, et al. Incident HIV during pregnancy and postpartum and risk of mother-to-child HIV transmission: a systematic review and meta-analysis. PLoS Med 2014;11:e1001608.

21 Graybill LA, Kasaro M, Freeborn K, et al. Incident HIV among pregnant and breast-feeding women in sub-Saharan Africa: a systematic review and meta-analysis. AIDS 2020;34:761-76.

22 WHO. Preventing HIV during pregnancy and breastfeeding in the context of prep. Geneva, Switzerland: WHO, 2017. http://www.who. int/hiv/pub/toolkits/prep-preventing-hiv-during-pregnancy/en

23 Baeten JM, Donnell D, Ndase P, et al. Antiretroviral prophylaxis for HIV prevention in heterosexual men and women. N Engl J Med 2012;367:399-410.

24 Grant RM, Lama JR, Anderson PL, et al. Preexposure chemoprophylaxis for HIV prevention in men who have sex with men. N Engl J Med Overseas Ed 2010;363:2587-99.

25 Pintye J, Beima-Sofie KM, Kimemia G, et al. "I Did Not Want to Give Birth to a Child Who has HIV": Experiences Using PrEP During Pregnancy Among HIV-Uninfected Kenyan Women in HIV-Serodiscordant Couples. J Acquir Immune Defic Syndr 2017;76:259-65.

26 Zimba C, Maman S, Rosenberg NE, et al. The landscape for HIV preexposure prophylaxis during pregnancy and breastfeeding in Malawi and Zambia: a qualitative study. PLoS One 2019;14:e0223487.

27 Pintye J, Drake AL, Kinuthia J, et al. A risk assessment tool for identifying pregnant and postpartum women who may benefit from preexposure prophylaxis. Clin Infect Dis 2017;64:751-8.

28 Sidebottom D, Ekström AM, Strömdahl S. A systematic review of adherence to oral pre-exposure prophylaxis for HIV - how can we improve uptake and adherence? BMC Infect Dis 2018;18:581.

29 Ministry of Health and Population, Malawi. Malawi population-based HIV impact assessment MPHIA 2015-2016. Available: https://phia. icap.columbia.edu/wp-content/uploads/2018/10/MPHIA-SS_2018 FINAL.pdf

30 Graybill L, Freeborn K, Kasaro M. A systematic review of risk factors for HIV acquisition during pregnancy and breastfeeding in subSaharan Africa. 10th International AIDS Society Conference on HIV Science 2019.

31 Hershow RB, Gonzalez M, Costenbader E, et al. Medical providers and harm reduction views on pre-exposure prophylaxis for HIV prevention among people who inject drugs. AIDS Educ Prev 2019;31:363-79.

32 Powers KA, Orroth K, Rosenberg NE. A mathematical modeling analysis of combination HIV prevention in antenatal clinics. In 2019 Conference on Retroviruses and Opportunistic Infections Seattle, WA 2019.

33 Amico KR, Miller J, Balthazar C, et al. Integrated next step counseling (iNSC) for sexual health and PreP use among young men who have sex with men: implementation and observations from ATN110/113. AIDS Behav 2019;23:1812-23.

34 R Amico K, McMahan V, Goicochea P, et al. Supporting study product use and accuracy in self-report in the iPrEx study: next step counseling and neutral assessment. AIDS Behav 2012;16:1243-59.

35 Berkman LF, Glass T. Social integration, social networks, social support, and health. Social epidemiology 2000;1:137-73.

36 InMinistry of Health and Population, Malawi. 2018 clinical management of HIV in children and adults. 4th ed. Lilongwe: Ministry of Health and Population, Malawi, 2018.

37 Doig GS, Simpson F. Randomization and allocation concealment: a practical guide for researchers. J Crit Care 2005;20:187-91.

38 Corneli AL, Deese J, Wang M, et al. FEM-PrEP: adherence patterns and factors associated with adherence to a daily oral study product for pre-exposure prophylaxis. J Acquir Immune Defic Syndr 2014;66:324-31.

39 Harris PA, Taylor R, Thielke R, et al. Research electronic data capture (REDCap)--a metadata-driven methodology and workflow process for providing translational research informatics support. $J$ Biomed Inform 2009:42:377-81. 
40 Chi BH, Cantrell RA, Zulu I, et al. Adherence to first-line antiretroviral therapy affects non-virologic outcomes among patients on treatment for more than 12 months in Lusaka, Zambia. Int J Epidemiol 2009;38:746-56.

41 Goldman JD, Cantrell RA, Mulenga LB, et al. Simple adherence assessments to predict virologic failure among HIV-infected adults with discordant immunologic and clinical responses to antiretroviral therapy. AIDS Res Hum Retroviruses 2008;24:1031-5.

42 World Health Organization. WHO announces COVID-19 outbreak a pandemic. Available: https://www.euro.who.int/en/health-topics/ health-emergencies/coronavirus-covid-19/novel-coronavirus-2019ncov

43 Chu DK, Akl EA, Duda S, et al. Physical distancing, face masks, and eye protection to prevent person-to-person transmission of SARS-
CoV-2 and COVID-19: a systematic review and meta-analysis. The Lancet 2020;395:1973-87.

44 Kinuthia J, Pintye J, Abuna F, et al. Pre-Exposure prophylaxis uptake and early continuation among pregnant and post-partum women within maternal and child health clinics in Kenya: results from an implementation programme. Lancet HIV 2020;7:e38-48.

45 The DHS Program ICF. Malawi demographic and health survey 2015-16, 2021. Available: https://dhsprogram.com/pubs/pdf/FR319/ FR319.pdf

46 LifeWindows Project Team. The LifeWindows information motivation behavioral skills art adherence questionnaire (LW-IMB-AAQ), 2006. Available: http://www.chip.uconn.edu/int/F_LWIMBARTQuestion naire.pdf 